\title{
IPSA Scientia
}

Artículo de Investigación

\section{Estudio descriptivo de la motivación del estudiante en cursos de matemáticas a nivel de educación superior}

\section{Descriptive study of student motivation in mathematics courses of higher education level}

\author{
ASTUDILLO-VILLALBA, Franklin \\ Instituto Superior de Formación Docente Salomé Ureña, San Juan de la Maguana, República Dominicana \\ TERÁN-BATISTA, Xavier \\ Instituto Superior de Formación Docente Salomé Ureña, San Juan de la Maguana, República Dominicana \\ DE OLEO-COMAS, Adrián \\ Instituto Superior de Formación Docente Salomé Ureña, San Juan de la Maguana, República Dominicana
}

Autor corresponsal: franklin.villalba@isfodosu.edu.do

Recibido: 27-04-2021; Aceptado: 23-08-2021; En línea: 31-08-2021

DOI: https://doi.org/10.25214/27114406.1112

Cómo citar este artículo:

Astudillo-Villalba, F., Terán-Batista, X., \& De Oleo-Comas, A.. (2021). Estudio descriptivo de la motivación del estudiante en cursos de matemáticas a nivel de educación superior. IPSA Scientia, revista científica multidisciplinaria, 6(3), 60-85. https://doi.org/10.25214/27114406.1112

Resumen - La motivación constituye una de las bases esenciales para la construcción del aprendizaje, el estudio de esta es de suma importancia para desarrollar procesos de enseñanza efectivos. En esta investigación, se evalúa el nivel de motivación en los estudiantes del Instituto Superior de Formación Docente Salomé Ureña para el estudio de las matemáticas que corresponden a su formación integral pedagógica. Para tal fin, se tomó una muestra de 172 alumnos correspondientes a los recintos con mayor población del instituto, los cuales se encuentran cursando la Licenciatura en Educación Primaria Segundo Ciclo. Posteriormente, se realizó una adaptación del cuestionario de Evaluación Motivacional del Proceso de Aprendizaje (EMPA), se sometió a validación por juicio de expertos y se aplicó la prueba del Coeficiente de Validez de Contenido (CVC). Luego, se realizó la prueba de fiabilidad del instrumento utilizando el Alfa de Cronbach. Después de la aplicación del instrumento, se determinó el nivel de satisfacción de la motivación individual y grupal, se hizo un análisis de veracidad vs contradicción con grupos de preguntas correlacionadas y se generaron hipótesis de las posibles variables que intervienen en la motivación ligada al aprendizaje de las matemáticas. En consecuencia, se observó que la mayoría de la población se encuentra en un nivel de satisfacción motivacional contradictorio o no definido, un segundo grupo se encuentra más motivado que desmotivado y finalmente, un alto porcentaje de los estudiantes tiene una clara veracidad en las respuestas de los ítems relacionados con la motivación intrínseca.

Palabras clave: Cuestionario, evaluación, matemáticas, motivación, proceso de aprendizaje.

Abstract - Motivation constitutes one of the essential bases for the construction of learning, its study is important to develop effective teaching processes. In this research, the level of motivation in the students of the Instituto Superior de Formación Docente Salomé Ureña is evaluated for the study of mathematics that correspond to their comprehensive pedagogical training. To carry out the research, a sample of 172 students corresponding to the 
institute's campuses with the highest population was taken, who are studying the Bachelor's Degree in Primary Education Second Cycle. Subsequently, an adaptation of the Motivational Assessment of the Learning Process (EMPA) questionnaire was carried out, it was subjected to validation by experts judgment and the Content Validity Coefficient (CVC) test was applied. Then, the instrument's reliability test was performed using Cronbach's Alpha. After the application of the instrument, the level of satisfaction of individual and group motivation was determined, an analysis of veracity vs contradiction was made with groups of correlated questions and hypotheses were generated of the possible variables that intervene in the motivation related to learning of the mathematics. Consequently, it was observed that the majority of the population is at a contradictory or undefined level of motivational satisfaction, a second group is more motivated than unmotivated and finally, a high percentage of students have a clear veracity in the answers of items related to intrinsic motivation.

Keywords: Questionnaire, evaluation, mathematics, motivation, learning process.

\section{Introducción}

Uno de los elementos más difíciles y complejos de diseñar e implementar en el proceso de enseñanza-aprendizaje es buscar por logro el mayor grado posible de aprendizaje en el alumnado(Quevedo-Blasco et al., 2016). Según Matos Matos (2019), diferentes estudios realizados por la Organización para la Cooperación y el Desarrollo Económico (OCDE) hay deserción del 30\% de los estudiantes universitarios. Citando a Garzón y Gil expresa que la deserción total universitaria en Latinoamérica se encuentra alrededor del 57\% de los estudiantes, siendo cada día más prominente en los estudiantes de los primeros semestres, dadas las dificultades del proceso de adaptación a un escenario complejo propio de la vida universitaria, tales como "la vocación profesional, la perspectiva de la misma, la formación primaria y los factores socio económicos” (Gutiérrez Echeverría, 2020, p. 208).

Zabala-Vargas et al. (2019), indican que hay poca información acerca de la reducción de las tasas de deserción (entendido como la no finalización de un programa académico). Si bien en general se acepta que existen múltiples causas, sugiere que los bajos resultados académicos y la baja motivación en un proceso educativo son causas importantes. En su investigación, realizada en su campo de estudio, expresó que el índice de deserción en los programas de ingeniería era aproximadamente del $45 \%$ y que el curso con las tasas de reprobación más altas era la matemática introductoria. En este contexto, propusieron estrategias y mediaciones pedagógicas innovadoras; emplearon métodos mixtos y de aprendizaje basado en juegos para diseñar un conjunto de estrategias didácticas. Para medir la motivación, aplicaron una adaptación de la Encuesta de Motivación del Material Instruccional, que les permitió medir las dimensiones de atención, confianza, satisfacción y relevancia. Ellos concluyen que la innovación pedagógica afecta positivamente la motivación de los estudiantes. Sus resultados más significativos incluyen la importancia de planificar actividades de aprendizaje rigurosas, incorporando problemas del entorno real del estudiante y la importancia de ofrecer retroalimentación inmediata. Esto indica que la motivación es una pieza fundamental que debe usar el docente en el aula durante todo el proceso a la hora de implementar sus actividades académicas.

Matos Matos (2019) expresa que "los modelos más recientes consideran a la motivación como un amplio constructo teórico e hipotético con diversas dimensiones, factores o variantes, más que una única variable observable y dependiente” (p. 2). 
Castro Navarro \& Ávila Contreras (2013), en su trabajo de investigación se enfocaron en el período de tiempo crítico en que algunos estudiantes deciden desertar de su carrera e indagan las experiencias y narraciones propias de los estudiantes. También concluye que "la motivación es un elemento crucial en el desempeño estudiantil. Si un sujeto está con una buena porción de motivación, su resiliencia se fortalece $y$, por ende, le da una suerte de recubrimiento para soportar distintos embates emocionales y afectivos” (p. 1294).

San Andrés-Soledispa et al. (2021), expresan que "la matemática es una materia de gran relevancia para la formación integral, pero despertar la motivación en esta área es muy difícil para los estudiantes del siglo XXI” (p. 671), por lo que es necesario modificar los sistemas y emplear nuevas estrategias para innovar. Siguiendo esta afirmación, Farias \& Pérez (2010) están de acuerdo que los docentes deben fortalecer su participación como orientadores en el proceso de enseñanza y de aprendizaje en las matemáticas, para que haya una transformación en la actitud de los estudiantes hacia está. En este sentido, concluye que "para lograr la motivación de los estudiantes se requiere conocer sus necesidades y expectativas para luego dirigir las conductas hacia acciones benéficas para los estudiantes y su contexto social” (p. 33). En concordancia con Gómez-Vahos et al. (2019), si se quiere lograr un aprendizaje significativo el docente debe

generar en el aula un ambiente que invite a todos a observar, investigar, a aprender, a construir su aprendizaje, y no sólo a seguir lo que él hace o dice. El rol del docente no debe ser solamente proporcionar información y controlar la disciplina, sino ser un mediador entre el estudiante y el ambiente, siendo el guía o acompañante del estudiante, mostrándole al estudiante que él es una gran fuente de conocimiento (pp. 120-121).

En la presente investigación se adaptó un cuestionario validado existente basado en la Evaluación Motivacional del Proceso de Aprendizaje (EMPA) establecido por Quevedo-Blasco et al. (2016), que tenga un excelente nivel de fiabilidad, aplicado con el objeto de evaluar el nivel de motivación que tienen los estudiantes de la Licenciatura en Educación Primaria Segundo Ciclo del Instituto Superior de Formación Docente Salomé Ureña para el estudio de las matemáticas.

\section{Referentes teóricos}

Calle Chacón et al. (2020), en su investigación para verificar la importancia de la motivación en el proceso de enseñanza-aprendizaje de las matemáticas, expresan que:

Hoy en día, un problema muy común que se puede observar en el área de matemática, es que los estudiantes demuestran cierta resistencia a desarrollar procesos de enseñanza aprendizaje, pues le produce miedo a no entender y por ende no poder solucionar los ejercicios con los que se encuentren, o en algunos casos, simplemente no les gusta la asignatura. Por otro lado, los docentes necesitan estar en formación continua para mejorar la forma en la que activan los conocimientos en clase, a través de actividades motivadoras, que animen el interés y la motivación de los estudiantes para aprender matemáticas (p. 490)

También indican que la motivación juega un papel importante y es uno de los factores fundamentales en el aprendizaje de las matemáticas, además de que el maestro debe fomentar en 
los estudiantes el placer de aprender. El fin no es que los estudiantes aprueben la asignatura, sino que lo hagan por el placer de construir su propio conocimiento matemático y aprender cosas nuevas. Según Fernández Paredes (2020), "la mayoría de los especialistas definen la motivación como un conjunto de procesos implicados en la activación, dirección y persistencia de la conducta” (p. 5). Luego expresa que en el contexto educativo "el estudiante implicará en este proceso las actitudes, percepciones y expectativas que tenga en cuanto a la tarea, a sí mismo y las metas que pretenda alcanzar” (p. 5).

De acuerdo con Álvarez Hernández \& Marín Rodríguez (2015), no todos los docentes se preocupan por la falta de motivación de los estudiantes en aprender, y en mayor grado aquellos del área de las matemáticas, la cual es una de las que más presenta desmotivación por parte de los estudiantes. En su investigación determinaron algunos factores que deben tenerse en cuenta para generar motivación en los estudiantes por aprender matemáticas, tales como la comodidad y la disposición del aula de clase, actividades que generen incentivos y competencia, las buenas relaciones interpersonales del docente ya que una mala actitud refuerza la apatía por el aprendizaje de las matemáticas. También manifiestan que "las matemáticas y su belleza son un factor motivacional porque son una interpretación del mundo y adicionalmente es un lenguaje universal que tiene las mismas representaciones gráficas en muchos idiomas” (p. 246).

Por otro lado, Mato Vázquez \& De la Torre Fernández (2010), expresan que la adquisición de ciertas habilidades matemáticas básicas y la comprensión de determinados conceptos son imprescindibles para un funcionamiento efectivo en la sociedad actual. Sin embargo, es frecuente observar la preocupación de muchos alumnos y profesores por el rendimiento inadecuado y por el rechazo y la apatía hacia la asignatura de matemáticas. En sus estudios lograron determinar que "la actitud hacia las matemáticas varía en función del tipo de centro. En este sentido se aprecian, la existencia de valores que van creciendo por este orden: público periferia, público centro, concertado y privado” (p. 205). Por lo cual, para que haya un cambio favorable en las actitudes del estudiante debe haber un mayor incremento en los conocimientos.

Mercader et al. (2017), opinan que "los enfoques actuales sobre el aprendizaje ponen de manifiesto igualmente la necesidad de integrar factores motivacionales en la explicación del rendimiento matemático" (p. 158).Desde esta perspectiva, mencionan que "una adecuada motivación es necesaria para la regulación de las estrategias cognitivas y metacognitivas que requiere un aprendizaje matemático significativo” (p. 158).

Gil Cuadra et al. (2017), afirman que "nadie discute la importancia de la alfabetización matemática en la formación de ciudadanos autónomos y críticos, sin embargo, en numerosas ocasiones, las matemáticas son vistas como algo tedioso y aburrido” (p. 85). Por tal motivo ratifica la importancia de la influencia de la motivación en el aprendizaje "cuando los estudiantes están concentrados y disfrutan (experimentan flujo) realizando actividades propuestas en el aula se implican más, invirtiendo más esfuerzo” (p. 85).

Zabala-Vargas et al. (2019) proponen una estrategia pedagógica, fundamentada en el aprendizaje basado en juegos-GBL, que permita incrementar la motivación de los estudiantes del área de matemáticas. Una conclusión similar contempla Terán-Batista \& De Oleo-Comas (2021) expresando que "la estrategia de aprendizaje basado en juegos utilizada de una manera adecuada 
por los docentes, ayuda a fortalecer las competencias específicas en el área de matemáticas” (p. 22). Pacheco-Carrascal (2016), expresa su opinión sobre la importancia que tiene la motivación, en el aprendizaje de las matemáticas respecto a la falta de entusiasmo que tienen los estudiantes y el poco deseo o interés de aprender al menos los conceptos básicos. También afirma que "la motivación es como un motorcito que nos impulsa, nos lleva y nos anima todo el tiempo a seguir la ruta trazada, con ahínco y coraje” (p. 150). Por tal razón, el docente debe motivar a los estudiantes y actuar como un motor impulsor para activar sus ánimos y deseos de superación, ya que ésta es parte de sus vidas y es un instinto natural.

Según Fernández Paredes (2020), se pueden apreciar tres dimensiones dentro de la motivación académica, como el componente del valor, el motivacional de la expectativa y el motivacional afectivo. Además, expresa que "los docentes deberán alentar a los estudiantes a desarrollar y aplicar estrategias para lograr la automotivación de forma que sean responsables de su propio aprendizaje a nivel metacognitivo, motivacional y conceptual” (p. 5). Además, Matos Matos (2019), citando a Fernández, Arnáiz, Mejías \& Barca (2015), manifiesta que:

Se ha demostrado que la motivación del rendimiento se incrementa en las situaciones en las que los alumnos atribuyen sus éxitos a factores internos y controlables, mientras que disminuye cuando dichas atribuciones se hacen a factores externos e incontrolables. Para mejorar la motivación de logro de los alumnos, y por tanto el proceso de aprendizaje, es importante que aquellos sepan atribuir tanto los éxitos como los fracasos al esfuerzo realizado, como causa interna, inestable y controlable por parte del sujeto (p. 4)

Castro \& Miranda (2019), reconocen que hace más de una década el interés de los docentes era muy escaso con respecto al estudio de la motivación en el aprendizaje de las matemáticas. También expresan que dentro de los factores afectivos que influyen en el aprendizaje de la matemática, la motivación es una variable que debe ser considerada; no obstante, este interés por su estudio ha crecido en los últimos años (Calle Chacón et al., 2020; Cardozo, 2019; Fernández Paredes, 2020; Llanga Vargas et al., 2019; Suninas \& Berciano, 2019; Fong Silva, Colpas Castillo \& Franco Borré, 2018).

De Sixte et al. (2020), precisan, tomando en cuenta el estudio de Pintrich y Schunk, que "la motivación intrínseca es una motivación humana innata que comienza en los niños pequeños como una necesidad indiferenciada de competencia y autodeterminación” (p. 68). También exponen que cuando la motivación es extrínseca esta puede variar en el grado en que se internaliza, pudiendo ser experimentada como más autónoma o más controlada.

Para Corredor-García \& Bailey-Moreno (2020), "la motivación extrínseca se genera en el exterior del estudiante y esto lo conduce a realizar una tarea determinada” (p. 133). Este tipo de motivación es más controlada por eventos externos, por ejemplo, sus estudiantes manifestaron que sus padres les prometen y otorgan algún tipo de recompensa o premio con la condición de que lleven a cabo sus responsabilidades académicas, se porten bien y obtengan buenas calificaciones. Con respecto a la motivación intrínseca expresan que "están relacionados con los motivos internos que impulsan a los estudiantes a alcanzar sus metas. Los estudiantes cuya motivación intrínseca es elevada tienen interés por aprender y disfrutan cuando realizan determinadas tareas” (p. 132). 
Riaño Garzón et al. (2018), plantean que los componentes y las características relacionadas con la motivación, surgen a partir de procesos intrínsecos y extrínsecos. De acuerdo a estos autores la motivación intrínseca es aquella donde el estudiante se motiva por la vivencia del proceso, más que por los logros o resultados; es decir, el estudiante estudia por el interés de la materia (Fong Silva, Curiel Gómez \& Brito Carrillo, 2017). Mientras que "la motivación extrínseca son aquellos estímulos externos que no guardan relación con la materia desarrollada y por ende generan en el estudiante la simple necesidad de aprobar el curso” (p. 361). Con referencia a esto, Castro \& Miranda (2019) afirman en sus resultados que no hubo indicios de que los estudiantes hayan estudiado matemáticas porque les guste la materia o por gusto personal. De hecho, sus resultados indicaron que estas motivaciones fueron exclusivamente de tipo extrínsecas.

\section{Materiales y Métodos}

Esta investigación asume un paradigma mixto debido a que integra parte cualitativa y cuantitativa de recolección de datos y análisis de la motivación de los estudiantes, que como lo expresa Ugalde Binda \& Balbastre Benavent (2013, p. 184), en este modelo los hallazgos son más completos, con una mayor confianza, mejor validación y entendimiento de los resultados. Además, el diseño es de tipo no experimental, de corte transversal y estudio instrumental.

La población tenida en cuenta para la investigación son los estudiantes del Instituto Superior de Formación Docente Salomé Ureña (ISFODOSU) de la República Dominicana, particularmente en los recintos Juan Vicente Moscoso (JVM), Luis Napoleón Núñez Molina (LNNM) y Urania Montás (UM) en el año 2020. La muestra de estudio estuvo compuesta por 172 estudiantes que han cursado asignaturas del área de matemáticas que se encuentran en el plan de estudio de la Licenciatura en Educación Primaria Segundo Ciclo a partir del segundo cuatrimestre del primer año de la carrera. En la Tabla 1y Tabla 2 se observa la distribución de la muestra por recintos y según el sexo respectivamente, y en esta última se evidencia una clara inclinación de las féminas por la educación primaria.

Tabla 1. División de la Muestra por Recintos

\begin{tabular}{ccc}
\hline Recinto & No. de estudiantes & Porcentaje \\
\hline JVM & 49 & 28.49 \\
LNNM & 31 & 18.02 \\
UM & 92 & 53.49 \\
\hline Total & 172 & 100 \\
\hline
\end{tabular}

Fuente: elaboración propia

Tabla 2. División de la Muestra por Sexo

\begin{tabular}{ccc}
\hline Sexo & No. de Estudiantes & Porcentaje (\%) \\
\hline $\mathrm{F}$ & 132 & 76.74 \\
$\mathrm{M}$ & 40 & 23.26 \\
\hline Total & 172 & 100 \\
\hline
\end{tabular}

Fuente: elaboración propia 
El muestreo utilizado es no probabilístico, en nuestro estudio fue por conveniencia, al elegir los recintos con la población existente en la carrera de educación primaria, por lo tanto, no es aleatorio, razón por la que se desconoce la probabilidad de selección de los elementos de la población debido a que no se eligieron los estudiantes al azar (Otzen \& Manterola, 2017).

El objetivo fundamental es evaluar el nivel de motivación que tienen los estudiantes de " $\mathrm{La}$ Pedagógica Dominicana” para el estudio de las matemáticas. Según Muñiz, Elosua \& Hambleton, citado por Matos Matos (2019):

Para la realización de este tipo de investigaciones, es fundamental disponer de instrumentos de evaluación, que permitan la confiabilidad y validez de la información recogida en los mismos. Para ello es necesario los estudios relacionados con la adaptación y validación de los test, los cuales han tenido notables incrementos en las últimas décadas, evidencia de su importancia como recursos imprescindibles en la toma de decisiones individuales o grupales (p. 4)

Para la recolección de los datos, se utilizó un instrumento tipo encuesta o cuestionario que fue elaborado tomando en cuenta el objetivo de la investigación y la variable motivación que fundamenta el estudio. Este se encuentra disponible en el Anexo 1.Tuapanta et al. (2017), afirman que "si bien los investigadores pueden optar por desarrollar su propio cuestionario, cabe indicar que esto es un proceso laborioso que puede llevar meses antes de conseguir una versión definitiva” (p. 38). También sugieren que otra alternativa es escoger o buscar instrumentos ya elaborados para recabar información. Rodríguez-Rodríguez \& Reguant-Álvarez (2020), afirman que si se utiliza un instrumento ya diseñado o implementado por otros autores y que se haya validado "es pertinente realizar los cálculos de fiabilidad dado que ésta puede variar según la población estudiada” (p. 4).

Previo a su aplicación a fin de determinar el grado de validez de su contenido, fue enviado para su validación a juicio de cinco expertos. Según Ruiz Bueno (2014), la validez de contenido se refiere a medir el concepto en cuestión durante el grado o el proceso de pasar de lo teórico a lo empírico. Escobar-Pérez \& Cuervo-Martínez (2008) afirman que, “el juicio de expertos se define como una opinión informada de personas con trayectoria en el tema, que son reconocidas por otros como expertos cualificados y que pueden dar información, evidencia, juicios y valoraciones" (p. 29). En concordancia con lo anterior, Escofet et al. (2016), expresan que "la validez de contenido es esencial a la hora de realizar inferencias o generalizaciones a partir de los resultados que obtendremos con el cuestionario" (p. 938).

Para la revisión de la redacción, los jueces consideraron los siguientes criterios: pertinencia, claridad y relevancia, valorando cada criterio de manera dicotómica (sí o no) donde: $\mathrm{Si}=1$ si hay ausencia de observaciones y No $=0$ si hay observaciones. Todos los jueces coincidieron en que el instrumento tenía pertinencia, claridad y relevancia.

Posteriormente, a manera de profundizar en la validez de contenido, se aplicó la concordancia de jueces a través de la técnica de proporción de acuerdos. Los niveles alcanzados para las dimensiones coherencia, pertinencia y claridad fue de 1, a pesar de que en pocos ítems algunos jueces mostraron no estar de acuerdo, esto no representó la mayoría. Los resultados son mayores 
a 0.70, concluyendo que el instrumento es válido según el juicio de expertos en todas las dimensiones (Ver Tabla 3, Tabla 4 y Tabla 5).

También, se aplicó el Coeficiente de Validez de Contenido (CVC), dado por Nieto Hernández (2002). Pedrosa et al. (2014), recomiendan, para este coeficiente, la participación de entre tres y cinco expertos; además al igual que otros coeficientes clásicos, éste permite valorar el grado de acuerdo a los expertos con cada uno de los ítems y al instrumento en general. Este CVC se calcula para cada elemento y luego se busca el promedio general del instrumento. Para esto se emplea la formula (1)

$$
\mathrm{CVC}_{i}=\frac{M_{x}}{V_{\text {máx }}}
$$

donde $M_{x}$ representa la media del reactivo dada por los jueces y $V_{\text {máx }}$ es la puntuación máxima que el reactivo puede alcanzar. Como la escala es dicotómica, el valor máximo es 1 y el mínimo es 0. También, para reducir el error o el posible sesgo dado por algún juez, es necesario calcular dicho error mediante la fórmula (2)

$$
\mathrm{Pe}_{i}=\left(\frac{1}{j}\right)^{j}
$$

donde $j$ es el número de jueces. Finalmente, el CVC se calcula aplicando la fórmula (3)

$$
\mathrm{CVC}=\mathrm{CVC}_{i}-\mathrm{Pe}_{i}
$$

luego, se toma el promedio de todos los ítems. Si es mayor a 0.7 se considera aceptable el instrumento. En efecto, aplicando este coeficiente, a todo el instrumento, se obtuvieron los siguientes índices 0.9892, 0.994 y 0.968 para las dimensiones coherencia, pertinencia y claridad respectivamente.

Por otro lado, Escobar-Pérez \& Cuervo-Martínez (2008) sustentan que: "El juicio de expertos se define como una opinión informada de personas con trayectoria en el tema, que son reconocidas por otros como expertos cualificados y que pueden dar información, evidencia, juicios y valoraciones” (p. 29).

En el mismo sentido, con el objetivo de tener la mejor validez mediante teorías especiales en el tema, se aplicó otro método de concordancia de jueces, mediante la prueba Binomial (4), la cual mide cuantitativamente el grado de acuerdos de los expertos en cada una de las dimensiones del contenido del cuestionario (coherencia, pertinencia y claridad). Si los resultados son menores a 0.05 se asume que el ítem y en consecuencia el instrumento posee validez de contenido. Los niveles alcanzados fueron respectivamente, 0.038, 0.038 y 0.027 , concluyendo que el instrumento es válido.

$$
P(x)=\left(\begin{array}{l}
n \\
x
\end{array}\right) p^{x}(1-p)^{n-x}, \text { con }\left(\begin{array}{l}
n \\
x
\end{array}\right)=\frac{n !}{x !(n-x) !}
$$


Tabla 3. Dimensión Coherencia

\begin{tabular}{|c|c|c|c|c|c|c|}
\hline Ítem & Juez 1 & Juez 2 & Juez 3 & Juez 4 & Juez 5 & Acuerdos \\
\hline 1 & 1 & 1 & 1 & 1 & 1 & 1 \\
\hline 2 & 1 & 1 & 1 & 1 & 1 & 1 \\
\hline 3 & 1 & 1 & 1 & 1 & 1 & 1 \\
\hline 4 & 1 & 1 & 1 & 1 & 1 & 1 \\
\hline 5 & 1 & 1 & 1 & 1 & 1 & 1 \\
\hline 6 & 1 & 1 & 1 & 1 & 1 & 1 \\
\hline 7 & 1 & 1 & 1 & 1 & 1 & 1 \\
\hline 8 & 1 & 1 & 1 & 1 & 1 & 1 \\
\hline 9 & 1 & 1 & 1 & 1 & 1 & 1 \\
\hline 10 & 1 & 1 & 1 & 1 & 1 & 1 \\
\hline 11 & 1 & 1 & 1 & 1 & 1 & 1 \\
\hline 12 & 1 & 1 & 1 & 1 & 0 & 1 \\
\hline 13 & 1 & 1 & 1 & 1 & 1 & 1 \\
\hline 14 & 1 & 1 & 1 & 1 & 1 & 1 \\
\hline 15 & 1 & 1 & 1 & 1 & 1 & 1 \\
\hline 16 & 1 & 1 & 1 & 1 & 1 & 1 \\
\hline 17 & 1 & 1 & 1 & 1 & 1 & 1 \\
\hline 18 & 1 & 1 & 1 & 1 & 1 & 1 \\
\hline 19 & 1 & 1 & 1 & 1 & 1 & 1 \\
\hline 20 & 1 & 1 & 1 & 1 & 1 & 1 \\
\hline 21 & 1 & 1 & 1 & 1 & 1 & 1 \\
\hline 22 & 1 & 1 & 1 & 1 & 0 & 1 \\
\hline 23 & 1 & 1 & 1 & 1 & 1 & 1 \\
\hline 24 & 1 & 1 & 1 & 1 & 1 & 1 \\
\hline 25 & 1 & 1 & 1 & 1 & 1 & 1 \\
\hline 26 & 1 & 1 & 1 & 1 & 1 & 1 \\
\hline 27 & 1 & 1 & 1 & 1 & 1 & 1 \\
\hline 28 & 1 & 1 & 1 & 1 & 1 & 1 \\
\hline 29 & 1 & 1 & 1 & 1 & 1 & 1 \\
\hline 30 & 1 & 1 & 1 & 1 & 1 & 1 \\
\hline 31 & 1 & 1 & 1 & 1 & 1 & 1 \\
\hline 32 & 1 & 1 & 1 & 1 & 1 & 1 \\
\hline 33 & 1 & 1 & 1 & 1 & 1 & 1 \\
\hline 34 & 1 & 1 & 1 & 1 & 1 & 1 \\
\hline 35 & 1 & 1 & 1 & 1 & 1 & 1 \\
\hline 36 & 1 & 1 & 1 & 1 & 1 & 1 \\
\hline 37 & 1 & 1 & 1 & 1 & 1 & 1 \\
\hline \multirow[t]{3}{*}{38} & 1 & 1 & 1 & 1 & 1 & 1 \\
\hline & & & & & Total & 38 \\
\hline & & & & & Índice & 1.0000 \\
\hline
\end{tabular}

Fuente: elaboración propia 
Tabla 4. Dimensión Pertinencia

\begin{tabular}{|c|c|c|c|c|c|c|}
\hline Ítem & Juez 1 & Juez 2 & Juez 3 & Juez 4 & Juez 5 & Acuerdos \\
\hline 1 & 1 & 1 & 1 & 1 & 1 & 1 \\
\hline 2 & 1 & 1 & 1 & 1 & 1 & 1 \\
\hline 3 & 1 & 1 & 1 & 1 & 1 & 1 \\
\hline 4 & 1 & 1 & 1 & 1 & 1 & 1 \\
\hline 5 & 1 & 1 & 1 & 1 & 1 & 1 \\
\hline 6 & 1 & 1 & 1 & 1 & 1 & 1 \\
\hline 7 & 1 & 1 & 1 & 1 & 1 & 1 \\
\hline 8 & 1 & 1 & 1 & 1 & 1 & 1 \\
\hline 9 & 1 & 1 & 1 & 1 & 1 & 1 \\
\hline 10 & 1 & 1 & 1 & 1 & 1 & 1 \\
\hline 11 & 1 & 1 & 1 & 1 & 1 & 1 \\
\hline 12 & 1 & 1 & 1 & 1 & 0 & 1 \\
\hline 13 & 1 & 1 & 1 & 1 & 1 & 1 \\
\hline 14 & 1 & 1 & 1 & 1 & 1 & 1 \\
\hline 15 & 1 & 1 & 1 & 1 & 1 & 1 \\
\hline 16 & 1 & 1 & 1 & 1 & 1 & 1 \\
\hline 17 & 1 & 1 & 1 & 1 & 1 & 1 \\
\hline 18 & 1 & 1 & 1 & 1 & 1 & 1 \\
\hline 19 & 1 & 1 & 1 & 1 & 1 & 1 \\
\hline 20 & 1 & 1 & 1 & 1 & 1 & 1 \\
\hline 21 & 1 & 1 & 1 & 1 & 1 & 1 \\
\hline 22 & 1 & 1 & 1 & 1 & 1 & 1 \\
\hline 23 & 1 & 1 & 1 & 1 & 1 & 1 \\
\hline 24 & 1 & 1 & 1 & 1 & 1 & 1 \\
\hline 25 & 1 & 1 & 1 & 1 & 1 & 1 \\
\hline 26 & 1 & 1 & 1 & 1 & 1 & 1 \\
\hline 27 & 1 & 1 & 1 & 1 & 1 & 1 \\
\hline 28 & 1 & 1 & 1 & 1 & 1 & 1 \\
\hline 29 & 1 & 1 & 1 & 1 & 1 & 1 \\
\hline 30 & 1 & 1 & 1 & 1 & 1 & 1 \\
\hline 31 & 1 & 1 & 1 & 1 & 1 & 1 \\
\hline 32 & 1 & 1 & 1 & 1 & 1 & 1 \\
\hline 33 & 1 & 1 & 1 & 1 & 1 & 1 \\
\hline 34 & 1 & 1 & 1 & 1 & 1 & 1 \\
\hline 35 & 1 & 1 & 1 & 1 & 1 & 1 \\
\hline 36 & 1 & 1 & 1 & 1 & 1 & 1 \\
\hline 37 & 1 & 1 & 1 & 1 & 1 & 1 \\
\hline \multirow[t]{3}{*}{38} & 1 & 1 & 1 & 1 & 1 & 1 \\
\hline & & & & & Total & 38 \\
\hline & & & & & Índice & 1.0000 \\
\hline
\end{tabular}

Fuente: elaboración propia 
Tabla 5. Dimensión Claridad

\begin{tabular}{|c|c|c|c|c|c|c|}
\hline Ítem & Juez 1 & Juez 2 & Juez 3 & Juez 4 & Juez 5 & Acuerdos \\
\hline 1 & 1 & 1 & 1 & 1 & 1 & 1 \\
\hline 2 & 1 & 1 & 1 & 1 & 0 & 1 \\
\hline 3 & 1 & 1 & 1 & 1 & 1 & 1 \\
\hline 4 & 1 & 1 & 1 & 1 & 1 & 1 \\
\hline 5 & 1 & 1 & 1 & 1 & 1 & 1 \\
\hline 6 & 1 & 1 & 1 & 1 & 1 & 1 \\
\hline 7 & 1 & 1 & 1 & 1 & 1 & 1 \\
\hline 8 & 1 & 1 & 1 & 1 & 1 & 1 \\
\hline 9 & 1 & 1 & 1 & 1 & 1 & 1 \\
\hline 10 & 1 & 1 & 1 & 1 & 0 & 1 \\
\hline 11 & 1 & 1 & 1 & 1 & 1 & 1 \\
\hline 12 & 1 & 1 & 1 & 1 & 0 & 1 \\
\hline 13 & 1 & 1 & 1 & 1 & 1 & 1 \\
\hline 14 & 1 & 1 & 1 & 1 & 0 & 1 \\
\hline 15 & 1 & 1 & 1 & 1 & 1 & 1 \\
\hline 16 & 1 & 1 & 1 & 1 & 1 & 1 \\
\hline 17 & 1 & 1 & 1 & 1 & 1 & 1 \\
\hline 18 & 1 & 1 & 1 & 1 & 1 & 1 \\
\hline 19 & 1 & 1 & 1 & 1 & 1 & 1 \\
\hline 20 & 1 & 1 & 1 & 1 & 0 & 1 \\
\hline 21 & 1 & 1 & 1 & 1 & 1 & 1 \\
\hline 22 & 1 & 1 & 1 & 1 & 0 & 1 \\
\hline 23 & 1 & 1 & 1 & 1 & 1 & 1 \\
\hline 24 & 1 & 1 & 1 & 1 & 1 & 1 \\
\hline 25 & 1 & 1 & 1 & 1 & 1 & 1 \\
\hline 26 & 1 & 1 & 1 & 1 & 1 & 1 \\
\hline 27 & 1 & 1 & 1 & 1 & 1 & 1 \\
\hline 28 & 1 & 1 & 1 & 1 & 1 & 1 \\
\hline 29 & 1 & 1 & 1 & 1 & 1 & 1 \\
\hline 30 & 1 & 1 & 1 & 1 & 1 & 1 \\
\hline 31 & 1 & 1 & 1 & 1 & 1 & 1 \\
\hline 32 & 1 & 1 & 1 & 1 & 1 & 1 \\
\hline 33 & 1 & 1 & 1 & 1 & 1 & 1 \\
\hline 34 & 1 & 1 & 1 & 1 & 1 & 1 \\
\hline 35 & 1 & 1 & 1 & 1 & 1 & 1 \\
\hline 36 & 1 & 1 & 1 & 1 & 1 & 1 \\
\hline 37 & 1 & 1 & 1 & 1 & 1 & 1 \\
\hline \multirow[t]{3}{*}{38} & 1 & 1 & 1 & 1 & 1 & 1 \\
\hline & & & & & Total & 38 \\
\hline & & & & & Índice & 1.0000 \\
\hline
\end{tabular}

Fuente: elaboración propia 
La confiabilidad tipo consistencia interna de un instrumento de medición según HernándezSampieri \& Mendoza (2018, p. 229), “es el grado en que un instrumento produce resultados consistentes y coherentes en la muestra o casos”. Para determinar el nivel de confiabilidad del instrumento, le fue aplicado el cuestionario a un grupo de 67 estudiantes del recinto Urania Montás que han cursado al menos una de las asignaturas superior a la del estudio de la investigación en la Licenciatura en Educación Primaria Segundo Ciclo. Para ello, se utilizó la prueba del Alfa de Cronbach, es preciso mencionar que esta se calcula mediante la fórmula dada en (5). Sin embargo, para este análisis y el de otros factores de la investigación se utilizó el software IBM SPSS Statistics versión 26.

$$
\alpha=\left[\frac{k}{k-1}\right]\left[1-\frac{\sum_{i=1}^{k} S_{i}^{2}}{S_{t}^{2}}\right]
$$

Donde $k$ es el número de preguntas de la encuesta, $S_{i}^{2}$ es la varianza del ítem $i$ y $S_{t}^{2}$ es la varianza de los valores totales observados. El criterio general utilizado para el análisis de los resultados de fiabilidad se muestra en la Tabla 6, de acuerdo con la sugerencia de George \& Mallery (2003, p. 231).

Tabla 6. Criterio del Alfa de Cronbach

\begin{tabular}{|c|c|}
\hline Intervalo & Conclusión \\
\hline $1-0.9$ & Excelente \\
\hline $0.89-0.8$ & Bueno \\
\hline $0.79-0.7$ & Aceptable \\
\hline $0.69-0.6$ & Cuestionable \\
\hline $0.59-0.5$ & Pobre \\
\hline Menor a 0.5 & Inaceptable \\
\hline
\end{tabular}

Además, dicha consistencia interna basada en fiabilidad según Campo-Arias \& Oviedo (2008), "se refiere al grado en que los ítems, puntos o reactivos que hacen parte de una escala se correlacionan entre ellos, la magnitud en que miden el mismo constructo” (p. 832). Por lo tanto, la consistencia interna aplicando el coeficiente de Alfa de Cronbach para escalas politómicas se considera fiable cuando se encuentra entre 0.70 y 1. En la Tabla 7 se observan los resultados obtenidos para el cuestionario de estudio:

Tabla 7. Análisis de Fiabilidad del Instrumento

\begin{tabular}{llrr}
\hline & \multicolumn{1}{c}{ N } & $\%$ \\
\hline \multirow{2}{*}{ Casos } & Válido & 67 & 100 \\
& Excluido & 0 & 0 \\
& Total & 67 & 100 \\
\cline { 2 - 3 } & Estadísticas de fiabilidad & \\
\cline { 2 - 3 } & Alfa de Cronbach Núm. de preguntas & \\
& 0.882 & 38 \\
\hline
\end{tabular}

Fuente: elaboración propia 
Como se observó, el nivel de fiabilidad es de 0.882 que para la escala total en la Tabla 6, constituye una confiabilidad buena. Por lo tanto, de acuerdo con los criterios de esta prueba, se determina que el cuestionario es consistente, por lo que la información obtenida es fiable. Posteriormente, se procede a realizar la aplicación del instrumento a toda la muestra seleccionada, posteriormente al obtener la data se realiza un análisis de veracidad del cuestionario a través de una adaptación de la técnica de Iadov, se determina el índice de satisfacción grupal, se analizan las respuestas de los estudiantes a las preguntas del cuestionario que están correlacionadas y se establecen las conclusiones del estudio.

\section{Resultados y Discusión}

Luego de realizar la interpretación de los datos arrojados por el cuestionario aplicado, se presenta el análisis y discusión de los resultados obtenidos. Cabe resaltar que en este estudio no se analizan los diferentes factores que causan algún nivel de motivación en los estudiantes; sin embargo, se generan hipótesis de las posibles variables que intervienen en la motivación del aprendizaje de las matemáticas de acuerdo con las respuestas al cuestionario. Fernández Sotelo \& Vanga Arvelo (2015), expresan que

A partir de la reflexión y criterio de otros investigadores y la experiencia investigativa personal, los autores emiten sus consideraciones sobre las particularidades de la aplicación de la Técnica de Iadov, como herramienta para evaluar el nivel de satisfacción o insatisfacción de individuos y grupos que han sido sometidos a procesos de cambio en su esfera de actuación, por la introducción de resultados científicos. (p. 180)

Para evaluar el nivel de satisfacción o nivel de veracidad del cuestionario, se hizo una modificación de la Técnica de Iadov, la cual determina el nivel de satisfacción de motivación individual y grupal a partir de una encuesta aplicada (Kuzmina, 1970).

Tabla 8. Adaptación del Cuadro Lógico de Iadov para Medir el Grado de Veracidad del Cuestionario

42. ¿Te gustaría, ahora mismo, estar haciendo otras cosas en lugar de estar en clase?

\begin{tabular}{ccccc}
\hline & \multicolumn{2}{c}{ No } \\
$\begin{array}{c}\text { 5. ¿Le gusta estudiar } \\
\text { matemáticas? }\end{array}$ & 26. Si pudieras escoger entre estudiar o no estudiar \\
matemáticas. ¿estudiarías?
\end{tabular}

Fuente: elaboración propia

En la Tabla 8, el número obtenido en la interrelación de las tres preguntas indica el estatus de cada estudiante en seis diferentes niveles de satisfacción en su motivación, mostradas en la Tabla 9 (Quevedo-Blasco et al., 2016). Por ejemplo, si un estudiante responde "Si" a la pregunta 42, se 
selecciona la zona derecha del cuadro donde aparece "Si". Si a la pregunta 26 responde "No" se selecciona el "No" que aparece debajo del "Si" anterior. Si a la pregunta 5 responde: "Poco" entonces se busca en la última columna de la tabla, la fila donde aparece dicha respuesta y se selecciona el punto donde se intercepta la fila "Poco" con la columna "No". El resultado del estudiante es “4” y equivale a "Más desmotivado que motivado".

Tabla 9. Niveles de Satisfacción Cuadro Lógico de Iadov

\begin{tabular}{cl}
\hline Nivel & Escala de satisfacción motivacional \\
\hline 1 & Clara motivación \\
2 & Más motivado que desmotivado \\
3 & Motivación no definida \\
4 & Más desmotivado que motivado \\
5 & Clara desmotivación \\
6 & Contradictorio \\
\hline
\end{tabular}

Fuente: elaboración propia

La aplicación de la encuesta a los estudiantes arrojó los siguientes resultados de satisfacción que se resumen en laTabla 10 y se representan mediante un diagrama de barras en la Figura 1.

Tabla 10. Porcentaje de Satisfacción de los Estudiantes Encuestados

\begin{tabular}{lc}
\hline \multicolumn{1}{c}{ Escala de satisfacción motivación } & Porcentaje \\
\hline Clara motivación & $6 \%$ \\
Más motivado que desmotivado & $29 \%$ \\
Motivación no definida & $24 \%$ \\
Más desmotivado que motivado & $2 \%$ \\
Clara desmotivación & $2 \%$ \\
Contradictorio & $38 \%$ \\
\cline { 2 - 2 } & $100 \%$ \\
\hline
\end{tabular}

Fuente: elaboración propia

Figura 1. Diagrama del Porcentaje Satisfacción de los Estudiantes Encuestados

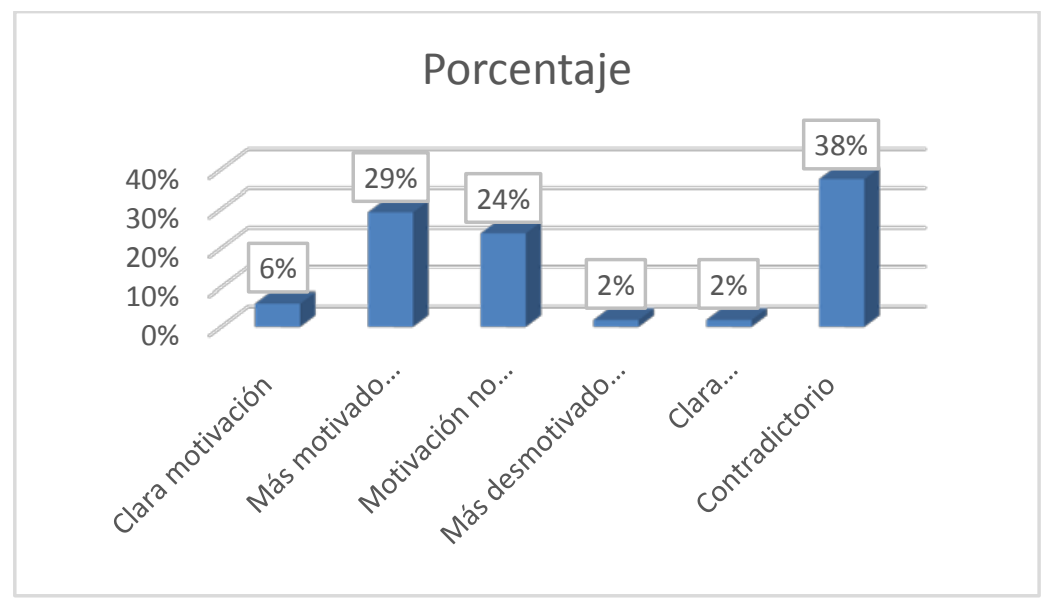

Fuente: elaboración propia 
Con estos datos, se puede obtener el índice de satisfacción de motivación grupal (ISMG) que según Quevedo-Blasco et al. (2016) maneja una escala numérica que oscila entre -1 y 1, tal como se puede ver en laTabla 11:

Tabla 11. Índice de Satisfacción de Motivacional Grupal (ISMG)

\begin{tabular}{ll}
\hline \multicolumn{1}{c}{ Puntuación } & Interpretación \\
\hline Máxima motivación & 1 \\
Más motivado que desmotivado & 0.5 \\
Motivación no definida y contradictoria & 0 \\
Más desmotivado que motivado & -0.5 \\
Máxima desmotivación & -1 \\
\hline
\end{tabular}

Fuente: elaboración propia

Además, dicha motivación grupal se calcula por la fórmula que se muestra a continuación en (6):

$$
I S M G=\frac{1 \times A+0.5 \times \mathrm{B}+0 \times \mathrm{C}-0.5 \times \mathrm{D}-1 \times \mathrm{E}}{N}
$$

donde A, B, C, D y E representan el número de estudiantes con índices 1, 2, 3 o 6, 4 y 5 respectivamente, y $N$ es el número total de los estudiantes. En nuestro estudio el ISMG $=0.18$ que se ubica en el rango de "Motivación no definida y contradictoria” (ver Figura 2).

Figura 2. Gráfica del Índice de Satisfacción Grupal

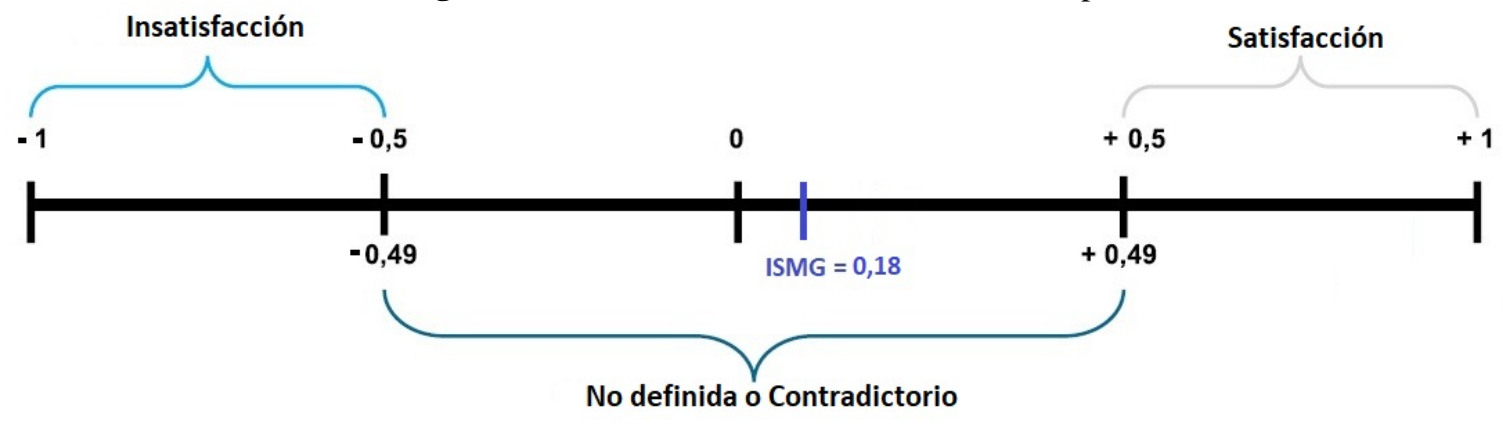

Fuente: elaboración propia

En la Figura 3, Figura 4 y Figura 5 que se muestran a continuación, se aprecia de manera resumida, los porcentajes de estudiantes que respondieron a las preguntas enmarcadas en la Tabla 8.

Figura 3. Respuestas a la Pregunta 42 de la Encuesta

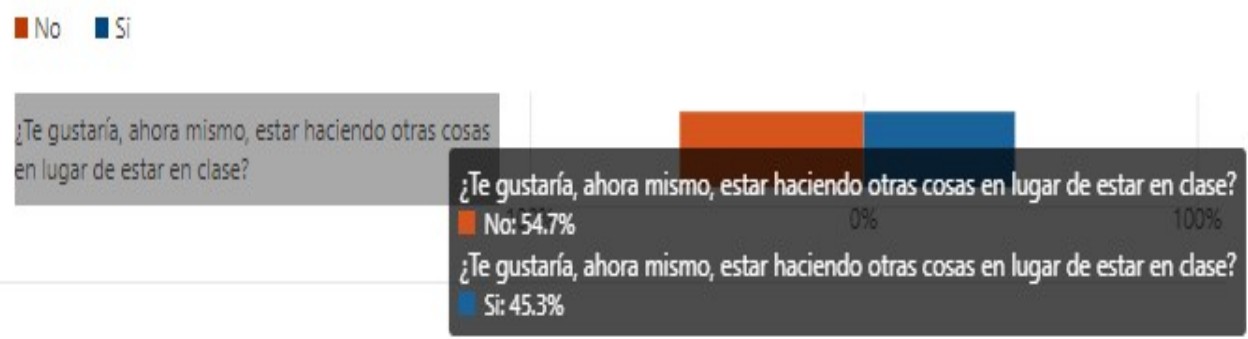

Fuente: elaboración propia 
Figura 4. Respuestas a la Pregunta 26 de la Encuesta

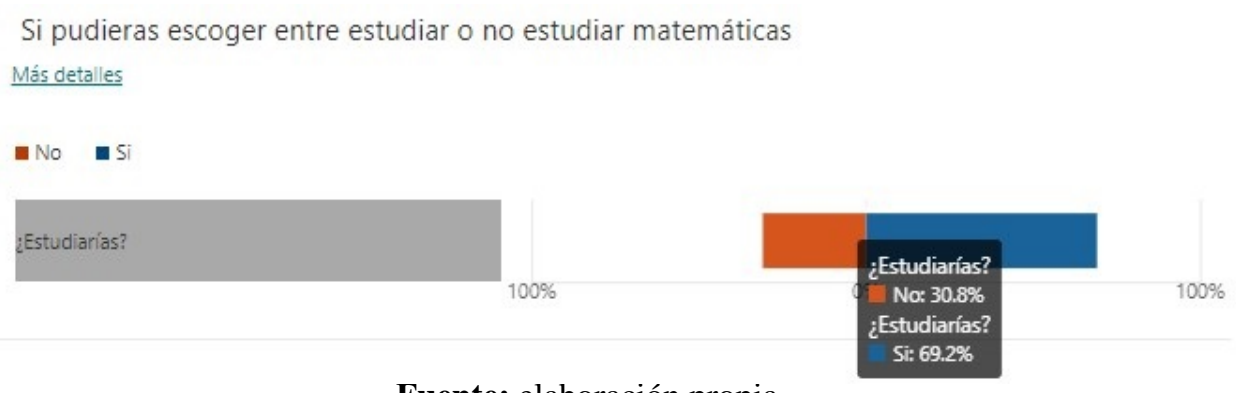

Fuente: elaboración propia

Figura 5. Respuestas a la Pregunta 5 de la Encuesta

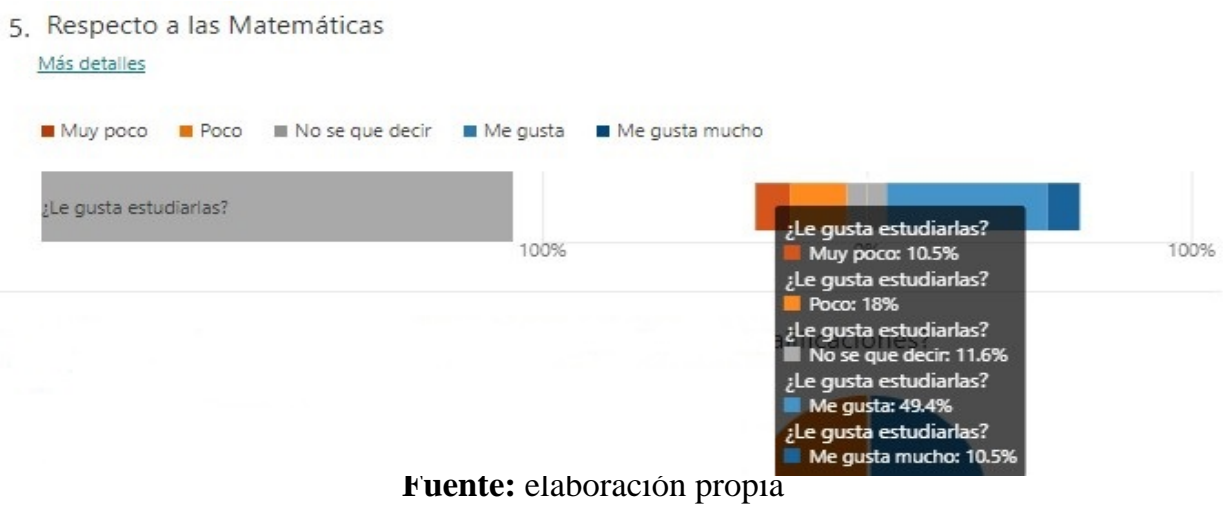

\section{Preguntas de Motivación Correlacionadas}

Adicionalmente, se ha realizado un análisis de veracidad vs contradicción con grupos de preguntas correlacionadas, con la finalidad de observar el contraste de las respuestas de los estudiantes con el porcentaje de satisfacción o índice de motivación. Para ello se toma en cuenta la escala según laTabla 12.

Tabla 12. Niveles de Veracidad de los Ítems Correlacionados

\begin{tabular}{ll}
\hline & Escala de veracidad - contradicción \\
\hline 1 & Clara veracidad \\
2 & Veracidad \\
3 & Veracidad no definida \\
4 & Contradicción \\
5 & Clara Contradicción \\
\hline
\end{tabular}

Fuente: elaboración propia

Es importante mencionar que el cuestionario tiene preguntas relacionadas con la motivación intrínseca y extrínseca de un estudiante, los ítems que conforman la motivación extrínseca son: 6, $7,10,12,13,15,16,17,23$ y 24, mientras que los encargados de evaluar la motivación intrínseca son: 5, 8, 9, 11, 14, 18, 19, 20, 21, 22 y de la 25 a la 42, teniendo en cuenta que las 
primeras cuatro preguntas corresponden a la información general de la muestra seleccionada para el estudio.

Las preguntas correlacionadas que se presentan en el cuestionario conforman dos grupos, el primer grupo está conformado por tres preguntas que evalúan la motivación extrínseca del estudiante, mientras que el segundo grupo lo conforman cinco preguntas encaminadas al estudio de la motivación intrínseca de la muestra seleccionada. A continuación, se describen de manera precisa cada una de estas preguntas mediante la Tabla 13.

Tabla 13. Grupos de Preguntas Correlacionadas del Cuestionario

\section{Preguntas}

\section{6. ¿Le preocupa lo que piensan de usted cuando saca malas calificaciones?}

Grupo 1

10. Cuando el profesor(a) pregunta en clase. ¿Le preocupa que sus compañeros(as) se rían de usted por no saber la respuesta?

17. ¿Le preocupa lo que el profesor(a) piensa de usted cuando no estudia?

18. ¿Es disciplinado en la asignatura para tomar mejores decisiones en la vida?

20. ¿Obtienes buenas calificaciones para tener un mejor futuro?

Grupo 2 25. ¿Estudias para ser mejor persona en la vida?

29. ¿Estudia y realiza tareas para resolver problemas que le surjan en la vida?

31. ¿Estudia para aprender a cambiar su forma de pensar y tener mejor estilo de vida?

\section{Fuente: elaboración propia}

Ahora, se realiza el análisis de la adaptación del cuadro lógico de Iadov, donde se interpreta el nivel de veracidad vs contradicción del grupo 1 de preguntas, observe la Tabla 14.

Tabla 14. Interpretación de los Ítems que Miden el Grado de Veracidad del Cuestionario Atendiendo a la Adaptación del Cuadro Lógico de Iadov

\begin{tabular}{|c|c|c|c|c|c|c|c|c|c|c|}
\hline \multirow{4}{*}{$\begin{array}{c}17 . \text { ¿Le preocupa lo que el } \\
\text { profesor(a) piensa de usted cuando } \\
\text { no estudia? }\end{array}$} & \multicolumn{10}{|c|}{ 6. ¿Le preocupa lo que piensan de usted cuando saca malas calificaciones? } \\
\hline & \multicolumn{5}{|c|}{ No } & \multicolumn{5}{|c|}{$\mathrm{Si}$} \\
\hline & \multicolumn{10}{|c|}{$\begin{array}{l}\text { 10. Cuando el profesor(a) pregunta en clase. ¿Le preocupa que sus } \\
\text { compañeros(as) se rían de usted por no saber la respuesta? }\end{array}$} \\
\hline & 尝 & ज氙 & 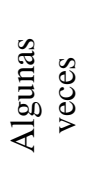 & 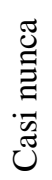 & $\underset{\Xi}{\Xi}$ & 营 & 矛 & 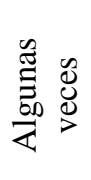 & 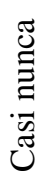 & $\underset{\Xi}{\Xi}$ \\
\hline Siempre & 5 & 5 & 5 & 5 & 5 & 1 & 2 & 2 & 3 & 3 \\
\hline Casi siempre & 5 & 5 & 4 & 4 & 4 & 2 & 2 & 2 & 4 & 4 \\
\hline Algunas veces & 4 & 4 & 3 & 3 & 3 & 3 & 3 & 3 & 4 & 4 \\
\hline Casi nunca & 4 & 4 & 2 & 2 & 2 & 4 & 4 & 4 & 5 & 5 \\
\hline Nunca & 3 & 3 & 2 & 2 & 1 & 5 & 5 & 5 & 5 & 5 \\
\hline
\end{tabular}

Fuente: elaboración propia 
Con relación a estas preguntas, el 39\% de los estudiantes indican en sus respuestas que no hay una veracidad definida, seguido de un $27 \%$ de ellos que al responder el cuestionario presentan una contradicción en la secuencia de respuestas que se presenta en la Tabla 14.

De la misma manera, en las tablas 15 y 16, se observa la adaptación para el grupo 2 de preguntas, teniendo en cuenta que, para este se toma como referencia el único ítem de este grupo con estilo de respuesta dicotómica y se analiza con cada par de preguntas con tipo de respuesta politómica, que realiza una subdivisión en Grupo 2-1 y Grupo 2-2.

Tabla 15. Interpretación de los Ítems que Miden el Grado de Veracidad del Cuestionario Grupo 2-1

\begin{tabular}{|c|c|c|c|c|c|c|c|c|c|c|}
\hline \multirow{4}{*}{$\begin{array}{l}\text { 20. ¿Obtienes buenas calificaciones para } \\
\text { tener un mejor futuro? }\end{array}$} & \multicolumn{10}{|c|}{ 25. ¿Estudias para ser mejor persona en la vida? } \\
\hline & \multirow{2}{*}{\multicolumn{10}{|c|}{$\begin{array}{l}\text { No } \\
\begin{array}{l}\text { 18. ¿Es disciplinado en la asignatura para tomar mejores decisiones } \\
\text { en la vida? }\end{array}\end{array}$}} \\
\hline & & & & & & & & & & \\
\hline & 蒙 & 氖 & $\begin{array}{l}\mathscr{Z} \\
\Xi \\
\Xi\end{array}$ & 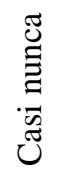 & 芯 & 节 & 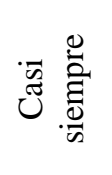 & 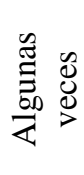 & 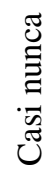 & 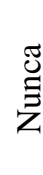 \\
\hline Siempre & 5 & 5 & 5 & 5 & 5 & 1 & 2 & 2 & 3 & 3 \\
\hline Casi siempre & 5 & 5 & 4 & 4 & 4 & 2 & 2 & 2 & 4 & 4 \\
\hline Algunas veces & 4 & 4 & 3 & 3 & 3 & 3 & 3 & 3 & 4 & 4 \\
\hline Casi nunca & 4 & 4 & 2 & 2 & 2 & 4 & 4 & 4 & 5 & 5 \\
\hline Nunca & 3 & 3 & 2 & 2 & 1 & 5 & 5 & 5 & 5 & 5 \\
\hline
\end{tabular}

Fuente: elaboración propia

Tabla 16. Interpretación de los Ítems que Miden el Grado de Veracidad del Cuestionario Grupo 2-2

\begin{tabular}{|c|c|c|c|c|c|c|c|c|c|c|}
\hline \multirow{4}{*}{$\begin{array}{l}\text { 31. ¿Estudia para aprender a cambiar su forma } \\
\text { de pensar y tener mejor estilo de vida? }\end{array}$} & \multicolumn{10}{|c|}{ 25. ¿Estudias para ser mejor persona en la vida? } \\
\hline & \multirow{2}{*}{\multicolumn{10}{|c|}{$\begin{array}{c}\text { No } \\
\text { 29. ¿Estudia y realiza tareas para resolver problemas que le } \\
\text { surjan en la vida? }\end{array}$}} \\
\hline & & & & & & & & & & \\
\hline & 范 & 苛 & 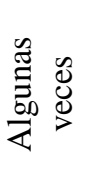 & 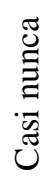 & $\begin{array}{l}\text { 己 } \\
\text { 吕 }\end{array}$ & 节 & 氙 & 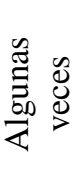 & 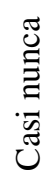 & 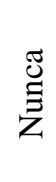 \\
\hline Siempre & 5 & 5 & 5 & 5 & 5 & 1 & 2 & 2 & 3 & 3 \\
\hline Casi siempre & 5 & 5 & 4 & 4 & 4 & 2 & 2 & 2 & 4 & 4 \\
\hline Algunas veces & 4 & 4 & 3 & 3 & 3 & 3 & 3 & 3 & 4 & 4 \\
\hline Casi nunca & 4 & 4 & 2 & 2 & 2 & 4 & 4 & 4 & 5 & 5 \\
\hline Nunca & 3 & 3 & 2 & 2 & 1 & 5 & 5 & 5 & 5 & 5 \\
\hline
\end{tabular}

Fuente: elaboración propia

De acuerdo a los datos anteriores para ambos casos, se analizan las preguntas relacionadas con la motivación intrínseca de los estudiantes, mediante la prueba de veracidad vs contradicción y con respecto a cada una de las tablas, se observó que un $71 \%$ de los estudiantes responde en base a 
los índices 1 y 2, lo que indica que existe una "Clara veracidad” en las respuestas que dieron los estudiantes a este grupo de preguntas correlacionadas.

\section{Conclusiones}

A manera de concluir, se logró evaluar el nivel de motivación para estudiar matemáticas en los estudiantes de La Pedagógica Dominicana, obteniendo de esta manera que aproximadamente un 59.9\% de los encuestados asegura que les gusta o les gusta mucho estudiar las matemáticas, lo que representa un alto porcentaje no esperado de acuerdo al programa de estudio. Respecto a si pudieran escoger entre estudiar matemáticas o no estudiarlas, un $69.2 \%$ opta por estudiarlas y en cuanto al hecho de saber si a los estudiantes les gustaría estar haciendo otras cosas en lugar de estar en clases de matemáticas, un $45.3 \%$ decide estar en clases de matemáticas, lo que en cuestiones de evaluación de la satisfacción motivacional para el proceso de aprendizaje de las matemáticas, muestra una pequeña diferencia entre quienes dicen que les gusta estudiar con escoger hacerlo y está más distante el deseo por estar en las clases de matemáticas en lugar de hacer otra cosa.

A pesar de los porcentajes obtenidos en las preguntas mencionadas, la mayor parte de la muestra correspondiente a un $62 \%$ se encuentra dentro de la unificación de las categorías de "Motivación Contradictoria y No definida”. Por lo tanto, es evidente la necesidad de trabajar con la población de estudio por medio de estrategias que ayuden al desarrollo de competencias actitudinales, las cuales deben fomentar el disfrute y aprendizaje de las matemáticas basándose en el enfoque constructivista de la enseñanza.

Asumiendo que dentro de otros factores que influyen en el rendimiento académico se puede encontrar la motivación, los conocimientos previos, el tipo de asignatura, los recursos con los que se cuenta en el aula, la carga académica, el tiempo de desarrollo de las actividades y otros indicadores más específicos que se pueden desprender de estos, es posible inferir que una gran mejora en la motivación del estudio de las matemáticas debe afrontar un cambio significativo en el aprendizaje y posteriormente en las calificaciones de los estudiantes, lo que supone un reto para el maestro de establecer y aplicar técnicas que permitan fortalecer la motivación de los estudiantes.

Por otro lado, es preciso mencionar que algo más de una tercera parte de la muestra se encuentra más motivada que desmotivada o presentan una clara motivación. Si bien esto no se traduce literalmente como un futuro buen desempeño académico, queda claro que constituye un público objetivo para comparar su rendimiento en proporción con los demás grupos que forman parte del estudio.

Es por esto que se recomienda que los esfuerzos docentes vayan enfocados en la realización de procesos didácticos donde se implementen estrategias de aprendizajes basadas en juegos, estos esfuerzos más que enfocarse en lo conceptual deben generar conciencia en los alumnos sobre la importancia del estudio de las matemáticas y cómo las mismas ayudan en la búsqueda de soluciones a problemáticas del día a día. De esta manera, las clases serían más interesantes, divertidas e incrementarían la motivación de los estudiantes para estudiar matemáticas. 
En cuanto a los grupos de preguntas correlacionadas que separan la motivación extrínseca de la intrínseca, un 39\% de los estudiantes respondieron con una veracidad no definida aquellas preguntas del grupo 1 que comprenden la motivación extrínseca, como se mostró en la Tabla 14. Esto permite inferir que cuando se tienen en cuenta los factores de carácter externo, como las ventajas que ofrece la actividad que se realiza, en este caso el hecho de aprender matemáticas, constituyendo un medio para llegar a un fin y no un fin en sí misma, no está contemplado por la mayoría de los estudiantes de la muestra

Por su parte, en cuanto a un estudiante que fija su interés por el trabajo, demostrando un papel activo en la consecución de sus fines, en este caso de estudiar la asignatura de matemáticas y tiene clara sus aspiraciones y metas. Hubo una clara representación de veracidad de acuerdo a las respuestas en las preguntas que evidencian su motivación intrínseca, ratificándose con un 71\% de las respuestas establecidas por los estudiantes, como se observó en la Tabla 15 y Tabla 16.

Finalmente, se concluye que es evidente el interés de la comunidad académica en estudiar la motivación de los estudiantes y mejor aún, dado los hallazgos de lugar, explorar nuevas estrategias didácticas innovadoras de aprendizaje orientado a las matemáticas, que permitan una mejoría tanto en el aprendizaje como en el rendimiento académico.

\section{Agradecimientos}

Este estudio ha sido patrocinado por el Instituto Superior de Formación Docente Salomé Ureña (ISFODOSU), mediante su aprobación en la convocatoria interna de proyectos de investigación del año 2019 con código de propuesta VRI-PI-01-2019-009, realizada al beneficiario Xavier Antonio Terán Batista junto a su equipo investigador.

\section{Referencias}

Álvarez Hernández, N. A., \& Marin Rodríguez, N. L. (2015). Factores de motivación para las clases de matemáticas. Encuentro Distrital de Educación Matemática EDEM, 2, $241-246$. http://funes.uniandes.edu.co/9888/

Calle Chacón, L. P., Garcia-herrera, D. G., Ochoa-encalada, S. C., \& Erazo-álvarez, J. C. (2020). La motivación en el aprendizaje de la matemática: Perspectiva de estudiantes de básica superior. Revista Arbitrada Interdisciplinaria KOINONIA, 5(1), 488-507. https://doi.org/http://dx.doi.org/10.35381/r.k.v5i1.794

Campo-Arias, A., \& Oviedo, H. C. (2008). Propiedades psicométricas de una escala: la consistencia interna. Revista de Salud Pública, 10, 831-839. http://www.scielo.org.co/scielo.php?script=sci_arttext\&pid=S0124$\underline{00642008000500015}$

Cardozo F, J. A. (2019). La motivación en la actualidad como técnica didáctica para el aprendizaje. Revista Atlante: Cuadernos de Educación $\quad$ y $\quad$ Desarrollo, https://doi.org/https://www.eumed.net/rev/atlante/2019/09/motivacion-tecnica-aprendizaje.html

Castro Navarro, E., \& Ávila Contreras, J. (2013). Motivación hacia la matemática, experiencia de estudiantes de un curso inicial de cálculo universitario. In Acta Latinoamericana de Matemática Educativa, pp. 1287-1295. http://funs.uniandes.edu.co/4309/

Castro, E. J., \& Miranda, I. (2019). Experiencias Desmotivacionales y Motivacionales de Estudiantes Varones de 
Ingeniería para Estudiar Matemáticas. El Caso de la Universidad Andrés Bello en Santiago de Chile. Formación Universitaria, 12(6), 83-92. https://doi.org/10.4067/s0718-50062019000600083

Corredor-García, M. S., \& Bailey-Moreno, J. (2020). Motivación y concepciones a las que alumnos de educación básica atribuyen su rendimiento académico en matemáticas. Revista Fuentes, 22(1), 127-141. https://doi.org/https://doi.org/10.12795/revistafuentes.2020.v22.i1.10

De Sixte, R., Jáñez, A., Ramos, M., \& Rosales, J. (2020). Motivación, Rendimiento en Matemáticas y Prácticas Familiares: un Estudio de su Relación en $1^{0}$ de Educación Primaria. Psicología Educativa, 26(1), 67-75. https://doi.org/https://doi.org/10.5093/psed2019a16

Escobar-Pérez, J., \& Cuervo-Martínez, Á. (2008). Validez de contenido y juicio de expertos: una aproximación a su utilización. Avances En Medición, 6(1), 27-36.https://dialnet.unirioja.es/servlet/articulo?codigo=2981181

Escofet, A., Folgueiras, P., Luna, E., \& Palou, B. (2016). Elaboración y validación de un cuestionario para la valoración de proyectos de aprendizaje-servicio. Revista Mexicana de Investigacion Educativa, 21(70), 929949. http://www.scielo.org.mx/scielo.php?script=sci_arttext\&pid=S1405-66662016000300929

Farias, D., \& Pérez, J. (2010). Motivación en la Enseñanza de las Matemáticas y la Administración. Formación Universitaria, 3(6), 33-40. https://doi.org/10.4067/s0718-50062010000600005

Fernández Paredes, L. (2020). Las tres dimensiones de la motivación. Campus Educación Revista Digital Docente, 17, 5-8. https://www.campuseducacion.com/revista-digital-docente/numeros/17/files/assets/basic-html/page$\underline{5 . h t m l \#}$

Fernández Sotelo, A., \& Vanga Arvelo, M. G. (2015). Satisfacción o insatisfacción con la introducción de resultados científicos. ¿Cómo valorarla? SATHIRI, 8, 180. https://doi.org/10.32645/13906925.405

Fong-Silva, W., Colpas-Castillo, F., \& Franco-Borré, D. (2018). Academic performance and its association with class attendance, intrinsic motivation and gender in engineering students. IPSA Scientia, revista científica multidisciplinaria, 3(1), 10-16. https://doi.org/10.25214/27114406.927

Fong-Silva, W., Curiel-Gómez, R., \& Brito-Carrillo, C. (2017). Aprendizaje significativo y su relación con la motivación intrínseca, escuela de procedencia y estrategias cognitivas en estudiantes de ingeniería. IPSA Scientia, revista científica multidisciplinaria, 2(1), 55-64. https://latinjournal.org/index.php/ipsa/article/view/909

George, D., \& Mallery, P. (2003). SPSS for Windows step by step: A simple guide and reference. 11.0 update. Pearson. https://wps.ablongman.com/wps/media/objects/385/394732/george4answers.pdf

Gil Cuadra, F., Torres Prados, T., \& Montoro Medina, A. B. (2017). Motivación en matemáticas de estudiantes de primaria. International Journal of Developmental and Educational Psychology, 1(1), 85-94. https://doi.org/10.17060/ijodaep.2017.n1.v1.901

Gómez-Vahos, L. E., Muriel-Muñoz, L. E., \& Londoño-Vásquez, D. A. (2019). El papel del docente para el logro de un aprendizaje significativo apoyado en las TIC. Encuentros, 17(02), 118-131. https://doi.org/https://www.redalyc.org/journal/4766/476661510011/

Gutiérrez-Echeverría, R. (2020). Caracterización de la gerencia en la deserción estudiantil en instituciones de educación superior del Atlántico, Colombia. IPSA Scientia, revista científica multidisciplinaria, 5(1), 207216. https://doi.org/10.25214/27114406.1035

Hernández-Sampieri, R., \& Mendoza, C. (2018). Metodología de la investigación. Las rutas cuantitativa, cualitativa y mixta. McGraw-Hill. https://virtual.cuautitlan.unam.mx/rudics/?p=2612

Hernández Sampieri, R., Fernández Collado, C., \& Baptista Lucio, M. del P. (2014). Metodología de la 
investigación (6ta. Ed.). McGrraw-Hill.. https://www.uca.ac.cr/wp-content/uploads/2017/10/Investigacion.pdf

Kuzmina, N. V. (1970). Metódicas investigativas de la actividad pedagógica. Leni.

Llanga Vargas, E. F., Silva Ocaña, M. A., \& Vistin Remache, J. J. (2019). Motivación extrínseca e intrìnseca en el estudiante. Revista Atlante: Cuadernos de Educación y Desarrollo, 9, 1-15. https://doi.org/https://www.eumed.net/rev/atlante/2019/09/motivacion-extrinseca-intrinseca.html

Maseda Fernández, M. del C. (2011). Estudio bibliográfico de la motivación en el aprendizaje de las matemáticas y propuesta de talleres aplicados a la vida real. Universidad Internacional de La Rioja. https://reunir.unir.net/handle/123456789/2173

Mato Vázquez, M. D., \& De la Torre Fernández, E. (2010). Evaluación de las actitudes hacia las matemáticas y el rendimiento académico. PNA. Revista de Investigación En Didáctica de La Matemática, 5(1), 197208.https://dialnet.unirioja.es/servlet/articulo?codigo $=3629028$

Matos Matos, A. G. (2019). Validación de la Escala de Motivación Académica y Atribuciones Causales, en estudiantes de la Universidad de Camagüey "Ignacio Agramonte Loynaz. En Facultad de Ciencias Sociales. Universidad Central “Marta Abreu” de Las Villas. https://dspace.uclv.edu.cu/handle/123456789/11820

Mercader, J., Presentación, M.-J., Siegenthaler, R., Molinero, V., \& Miranda, A. (2017). Motivación y rendimiento académico en matemáticas: un estudio longitudinal en las primeras etapas educativas. Revista de Psicodidáctica, 22(2), 157-163. https://doi.org/10.1016/j.psicod.2017.05.007

Nieto Hernández, R. (2002). Contribuciones al análisis estadístico. Revista Venezolana de CIENCIA POLÍTICA, 23, 119. http://www.revencyt.ula.ve/storage/repo/ArchivoDocumento/cipo/v23/articulo10.pdf

Otzen, T., \& Manterola, C. (2017). Técnicas de Muestreo sobre una Población a Estudio. International Journal of Morphology, 35(1), 227-232. https://doi.org/10.4067/S0717-95022017000100037

Pacheco-Carrascal, N. (2016). La motivación y las matemáticas. Revista Ecomatemático, 7(1), 149-158. https://doi.org/10.22463/17948231.1026

Pedrosa, I., Suárez-álvarez, J., \& García-Cueto, E. (2014). Evidencias sobre la validez de contenido: avances teóricos y métodos para su estimación. ACCIÓN PSICOLÓGICA, 10(2), 3-20. https://doi.org/10.5944/ap.10.2.11820

Quevedo-Blasco, R., Quevedo-Blasco, V., \& Téllez-Trani, M. (2016). Cuestionario de evaluación motivacional del proceso de aprendizaje (EMPA). EJIHPE: European Journal of Investigation in Health, Psychology and Education, 6(2), 83-105. https://doi.org/10.1989/ejihpe.v6i2.161

Riaño Garzón, M. E., Torrado Rodríguez, J. L., Díaz Camargo, É. A., Vargas Martínez, D. E., Jiménez Jiménez, W. A., Durán Rodríguez, J. M., \& Rosero Reyes, G. P. (2018). Innovación Psicológica: Salud, Educación y Cultura. Ediciones Universidad Simón Bolívar. https://doi.org/https://www.researchgate.net/publication/331907838_Capitulo_XIV__Propiedades_Psicometricas_del_Instrumento_Motivacion_Intrinseca_y_Extrinseca_en_estudiantes_de_un_C olegio_de_Puerto_Santander

Rodríguez-Rodríguez, J., \& Reguant-Álvarez, M. (2020). Calcular la fiabilidad de un cuestionario o escala mediante el SPSS: el coeficiente alfa de Cronbach. REIRE Revista d'Innovació i Recerca En Educació, 13(2), 1-13. https://doi.org/10.1344/reire2020.13.230048

Ruiz Bueno, A. (2014). La operacionalización: de elementos teóricos al proceso de medida. Barcelona: Colección Omado. http://hdl.handle.net/2445/53152

San Andrés-Soledispa, E. J., San Andrés-Soledispa, E. M., \& Pazmiño-Campuzano, M. F. (2021). La gamificación 
como estrategia de motivación en la enseñanza de la asignatura de Matemática Gamification. Polo Del Conocimiento, 6(2), 670-685. https://doi.org/10.23857/pc.v6i2.2303

Suninas, A., \& Berciano, A. (2019). La motivación en el aula de matemáticas: ejemplo de Yincana $5^{\circ}$ de Educación Primaria. Números, Revista de Didáctica de Las Matemáticas, 101, $45-58$. https://doi.org/https://dialnet.unirioja.es/servlet/articulo?codigo=7053211

Terán-Batista, X., \& De Oleo-Comas, A. (2021). Enseñanza de permutaciones a estudiantes de educación superior mediante el uso de un juego clásico. IPSA Scientia, revista científica multidisciplinaria, 6(2), $10-25$. https://doi.org/10.25214/27114406.1062

Toledo Morales, P., \& Sánchez García, J. M. (2015). Diseño y validación de cuestionarios para percibir el uso de la pizarra digital interactiva (PDI) por docentes y estudiantes. Píxel-Bit, Revista de Medios y Educación, 47, 179-194. https://doi.org/10.12795/pixelbit.2015.i47.12

Tuapanta, J., Duque, M., \& Mena, Á. (2017). Alfa de Cronbach para validar un instrumento de uso de TIC en docentes universitarios. MktDESCUBRE, 10(141), 37-48. https://doi.org/10.36779/mktdescubre.v10.141

Ugalde Binda, N., \& Balbastre Benavent, F. (2013). Investigación Cuantitativa e Investigación Cualitativa: Buscando Las Ventajas De Las Diferentes Metodologías De Investigación. Revista de Ciencias Económicas, 31(2), 179-187. https://revistas.ucr.ac.cr/index.php/economicas/article/view/12730

Zabala-Vargas, S. A., Garcia-Mora, L. H., Ardila-Segovia, D. A., \& De Benito-Crosetti, B. L. (2019). Motivation increase of mathematics students in Engineering-A proposal from Game Based Learning. International Symposium on Engineering Accreditation and Education (ICACIT). https://doi.org/10.1109/ICACIT46824.2019.9130297

Zabala-Vargas, S., García-Mora, L., \& Benito-Crosetti, B. (2019). Estrategia pedagógica con aprendizaje basado en juegos-GBL, para fomentar la motivación en el área de matemáticas en ingeniería. In Estrategias didácticas para la innovación en la sociedad del conocimiento. http://memoriascimted.com/libros/ 


\section{ANEXO 1 \\ Estudio descriptivo de la motivación global en cursos de matemáticas}

Instrucciones

El siguiente es un cuestionario tipo encuesta que tiene como objetivo estudiar el nivel de motivación que tienen los estudiantes de la Licenciatura en Educación Primaria Segundo Ciclo del Instituto Superior de Formación Docente Salomé Ureña para el estudio de las matemáticas. Le garantizamos el absoluto anonimato de sus respuestas en el más estricto cumplimiento de la Leyes sobre secreto estadístico y protección de datos personales. (Aclaramos que esto no les afectará en sus calificaciones de promoción de grado, por tanto, no es necesario que agreguen sus nombres a este instrumento).

En el cuestionario se respetará cada una de las palabras que usted exprese y por concepción ética del investigador, no se omitirá ni se alterará cualquier información que facilite.

- Para no ser redundante, cabe resaltar que cada una de las preguntas mostradas en los ítems están realizadas en torno a la asignatura de matemáticas.

- Sea objetivo en sus respuestas.

- Seleccione la casilla que mejor corresponda con tu situación y recuerde que solo debes marcar una sola respuesta en cada una de las preguntas que así lo requieran.

- Se agradece en lo posible, responder todas las preguntas.

- Si tiene duda en algún planteamiento, consulte al Equipo Investigador.

1. ¿En cuál de las siguientes asignaturas se encuentra inscrito?
a. Matemática II
b. Matemática III
c. Didáctica especial de la matemática I
d. Didáctica especial de la matemática II
e. Matemática IV
f. Matemática V
g. He culminado los cursos de matemática

2. Seleccione el recinto del ISFODOSU al que pertenece:
a. Félix Evaristo Mejía (FEM)
b. Juan Vicente Moscoso (JVM)
c. Luis Napoleón Núñez Molina (LNNM)
d. Urania Montás (UM)

3. En que rango de los presentados se encuentra su edad
a. $16-18$
b. $19-21$
c. mayor a 22

4. Sexo
a. Femenino
b. Masculino

5. Respecto a las Matemáticas: ¿Le gusta estudiarlas?
a. Muy poco
b. Poco
c. No sé qué decir
d. Me gusta
e. Me gusta mucho

6. ¿Le preocupa lo que piensan de usted cuando saca malas calificaciones?
a. $\mathrm{Si}$
b. No 


\begin{tabular}{|c|c|c|c|c|c|}
\hline & $\begin{array}{l}\stackrel{\Xi}{E} \\
\text { 之 }\end{array}$ & 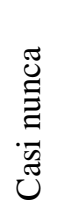 & 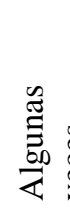 & 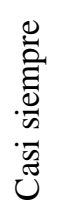 & 蒙 \\
\hline 7. ¿Intenta ser buen estudiante para que sus compañeros hablen bien de usted? & & & & & \\
\hline 8. ¿Estudia y presta atención en clase para mejorar su rendimiento académico? & & & & & \\
\hline $\begin{array}{l}\text { 9. ¿Al culminar la jornada de clases, lo primero que realizo son los deberes } \\
\text { académicos para disponer de mayor tiempo libre? }\end{array}$ & & & & & \\
\hline $\begin{array}{l}\text { 10. Cuando el profesor(a) pregunta en clase. ¿Le preocupa que sus compañeros(as) } \\
\text { se rían de usted por no saber la respuesta? }\end{array}$ & & & & & \\
\hline 11. ¿Cuándo obtiene buenas calificaciones continúa esforzándose en sus estudios? & & & & & \\
\hline $\begin{array}{l}\text { 12. ¿Estudia y realiza las tareas porque le gusta la pasión que el(a) profesor(a) } \\
\text { muestra en la asignatura? }\end{array}$ & & & & & \\
\hline 13. ¿Sientes satisfacción al sacar buenas calificaciones? & & & & & \\
\hline $\begin{array}{l}\text { 14. ¿Estudia y realiza las tareas para aprender a resolver los problemas que el } \\
\text { profesor(a) asigna en clase? }\end{array}$ & & & & & \\
\hline 15. ¿Estudia y realiza las tareas para que el profesor lo tome en cuenta? & & & & & \\
\hline 16. ¿Le gusta que el profesor(a) lo felicite por ser buen estudiante? & & & & & \\
\hline 17. ¿Le preocupa lo que el profesor(a) piensa de usted cuando no estudia? & & & & & \\
\hline 18. ¿Es disciplinado en la asignatura para tomar mejores decisiones en la vida? & & & & & \\
\hline 19. ¿En la asignatura, le gusta y le divierte aprender? & & & & & \\
\hline 20. ¿Obtienes buenas calificaciones para tener un mejor futuro? & & & & & \\
\hline 21. ¿Realiza las tareas porque le gusta ser responsable? & & & & & \\
\hline 22. ¿Aprende más cuando el profesor(a) coloca problemas difíciles? & & & & & \\
\hline $\begin{array}{l}\text { 23. ¿Estudia y realiza las tareas para que su profesor(a) lo considere un buen } \\
\text { alumno(a)? }\end{array}$ & & & & & \\
\hline $\begin{array}{l}\text { 24. ¿Estudia más cuando el profesor(a) utiliza herramientas tecnológicas y } \\
\text { materiales didácticos para desarrollar la clase? }\end{array}$ & & & & & \\
\hline
\end{tabular}

25. Si pudieras escoger entre estudiar o no estudiar matemáticas: ¿Estudiarías?

26. ¿Estudias para ser mejor persona en la vida? 
39. ¿Está desubicado "en las nubes” durante la jornada de clases?

40. ¿Desea con frecuencia que la jornada de clases termine pronto?

41. ¿Habitualmente participa en las discusiones o actividades que se realizan en clase?

42. Respecto a las matemáticas: ¿Te gustaría, ahora mismo, estar haciendo otras cosas en lugar de estar en clase?
a. $\mathrm{Si}$
b. No 